\title{
Minimally invasive removal of facial foreign body granulomas
}

Jaeik Choi ${ }^{1}$, Geonil Ko ${ }^{1}$, Hyeokjae Kwon ${ }^{1}$, Yooseok $\mathrm{Ha}^{1,2}$, Sunje Kim ${ }^{1}$, Hyunwoo Kyung ${ }^{3}$, Sang-Ha $\mathrm{Oh}^{1,2}$, Seung Han Song ${ }^{1,2}$

${ }^{1}$ Department of Plastic and Reconstructive Surgery, Chungnam National University Hospital, Daejeon; ${ }^{2}$ Department of Plastic and Reconstructive Surgery, Chungnam National University College of Medicine, Daejeon; ${ }^{3}$ Department of Plastic and Reconstructive Surgery, Chungnam National University Sejong Hospital, Sejong, Korea

This work was supported by the research fund of Chungnam National University.
Background Various materials, commonly called fillers, have been developed and are now used for cosmetic and reconstruction purposes. Indiscriminate injections of illegal and unknown substances have various side effects, among which foreign body granulomas are particularly difficult to treat. Surgical resection can be considered for small and well-defined foreign body granulomas, but complete resection is often impossible for wide facial granulomas, and postoperative deformities may occur. Therefore, this study presents cases where foreign bodies were evaluated using imaging studies and removed through minimally invasive procedures depending on their characteristics. Methods Thirty-five patients with chronic granulomas after illegal filler injections treated from 2012 to 2019 were enrolled. Clinically, these granulomas were classified into cystic and infiltrating groups according to the imaging study patterns. Patients in the cystic group underwent puncture and drainage, and those in the infiltrating group were first treated with intralesional laser treatment and then suctioned. If the results were insufficient, surgical removal combined with a lifting procedure was performed. Results All 35 patients were women, and their average age was 51 years. Surgery was successful in almost all cases, but four cases of insufficient removal and contour deformity were encountered during follow-up. Two patients underwent reoperation and two patients improved naturally.

Conclusions In this study, we classified the characteristics of granulomas using preoperative imaging studies. Aesthetically favorable results were obtained using puncture and drainage and tumescent suction, along with, if necessary, surgical removal accompanied by a lifting procedure when removing facial foreign body granulomas.

Keywords Foreign bodies / Granuloma / Injections / Ultrasonography / Cosmetics

\section{INTRODUCTION}

Since the introduction of fat grafts by the German physician Dr. Neuber in 1893, various substances such as paraffin, liquid silicone,

Received: Nov 8, 2021 Revised: Dec 26, 2021 Accepted: Jan 19, 2022 Correspondence: Seung Han Song Department of Plastic and Reconstructive Surgery, Chungnam National University Hospital, Chungnam National University College of Medicine, 282 Munhwa-ro, Jung-gu, Daejeon 35015 , Korea

Tel: +82-42-280-7380, Fax: +82-42-280-7384, E-mail: silverwine_@naver.com Copyright @ 2022 The Korean Society for Aesthetic Plastic Surgery.

This is an Open Access article distributed under the terms of the Creative Commons Attribution Non-Commercial License (https://creativecommons.org/licenses/by-nc/4.0/) which permits unrestricted non-commercial use, distribution, and reproduction in any medium, provided the original work is properly cited. www.e-aaps.org collagen, and hyaluronic acid have been developed and used for cosmetic and reconstructive purposes [1]. These substances are collectively called fillers, and since they can be administered by simple injection, patients' fear of surgery and anesthesia is reduced. Thus, filler injections are popular with patients because they enable a rapid return to daily life after the procedure. These procedures are widely used to improve wrinkles around the forehead, eyes, mouth, and cheeks in middle-aged women.

However, the frequency of indiscriminate injections of illegal unknown substances other than medical fillers for cosmetic purposes is increasing and becoming a social problem. The side effects that can occur after illegal filler procedures include acute side effects such as redness, erythema, abscess formation, non-specific inflammation, skin necrosis, ischemic damage, chronic nodules, and granuloma formation, and systemic reactions [2]. In particu- 
lar, it is difficult to treat chronic nodules, granulomas, and foreign body reactions several years after the procedure. Treatments include surgical resection for small lesions with clear boundaries. However, if the affected area is wide and the boundary is unclear, local or systemic steroids and empirical antibiotics can be used [3]. The effects of conservative medications such as steroids are often temporary; therefore, patients repeatedly experience lesion exacerbation and improvement, and often undergo surgical treatment due to worsening inflammation or infection. When unknown substances are widely injected into the forehead or midface and then removed, complete surgical removal is often impossible, and complications such as postoperative deformities are also likely to occur after resection [4].

Furthermore, recent reports have described good results after drainage, dead tissue resection using Versajet (Versajet hydrosurgery system; Smith \& Nephew, London, UK) and bipolar high-frequency devices (Innofill; Pacific Pharma, Seoul, Korea), which provide alternative treatments that overcome the limitations of surgical resection [5-7]. However, based on the authors' experience, the effects of those treatments are less than those of surgical resection.

In this study, we classified granulomas based on imaging study findings prior to removing foreign substances associated with chronic granulomas caused by injecting unknown substances into the face, such as the forehead and midface, and describe the outcomes achieved by intralesional laser treatment, and when necessary by surgical removal through an inconspicuous incision.

\section{METHODS}

This clinical study was conducted with the approval of the Institutional Review Board (IRB No. CNUH 201-10-063). Thirty-five patients who visited the hospital for chronic granulomas arising after unknown substance injections from May 2012 to May 2019 and subsequently underwent a removal procedure or surgery were included. All were women, and their average age was 51.2 years (range, 33-74 years). The minimum follow-up period was 1 month and the maximum was 36 months; the average follow-up was 15 months. Most patients were checked preoperatively using ultrasonography to determine the nature, quantity, and location of facial foreign body granulomas in lesions and degrees of inflammatory reactions. Filler granulomas were classified as cystic or infiltrating according to the image findings and treated on an individual basis. Pictures of patients were taken before surgery, during surgery, after surgery, at the first outpatient visit, and at the final follow-up visit to confirm cosmetic and functional results. Patients were monitored for complications until the end of treatment.

According to ultrasound patterns, patients were classified into a cystic group (group I; $n=7$ ), which showed a hypo-echoic or nonechoic lesions with a cystic morphology, and an infiltrating group (group II; $\mathrm{n}=28$ ), in which the lesions were iso-echogenic with normal tissues and foreign substances were scattered in surrounding tissues. In the cystic group, drainage was performed after a puncture. In the infiltrating group, after intralesional laser treatment, suction was performed that included some surrounding soft tissue (group IIA; $\mathrm{n}=14$ ). Surgical removal was performed in the infiltrating group when a foreign body was insufficiently removed by suction. To minimize the resulting deformity, such as depression, foreign body removal and pretrichial forehead lift were concomitantly performed (group IIB; $n=14$ ) (Fig. 1).

In most patients, physical examinations and ultrasonography were performed before the procedure or surgery using a 5-12 MHz linear transducer of a Hitachi Aloka ultrasound scanner (Hitachi Medical Systems, Twinsburg, OH, USA) to determine the foreign body location, size, and degree of skin depression. Cross-sectional and longitudinal grayscale images of lesions were obtained without compressing the lesion sites. Sleep anesthesia (midazolam and ketamine) was used during foreign substance removal, and when patients fell asleep, a local anesthetic ( $2 \%$ lidocaine) was injected evenly around the surgical site. The operations were performed as follows.

\section{Cystic group (group I)}

After puncture along the relaxed skin tension line using an 18-gauge needle, drainage was performed through manual compression after puncture, and the empty space was then washed with saline to remove residues that had adhered to the false film. If there was no evidence of infection, the puncture site was then repaired.

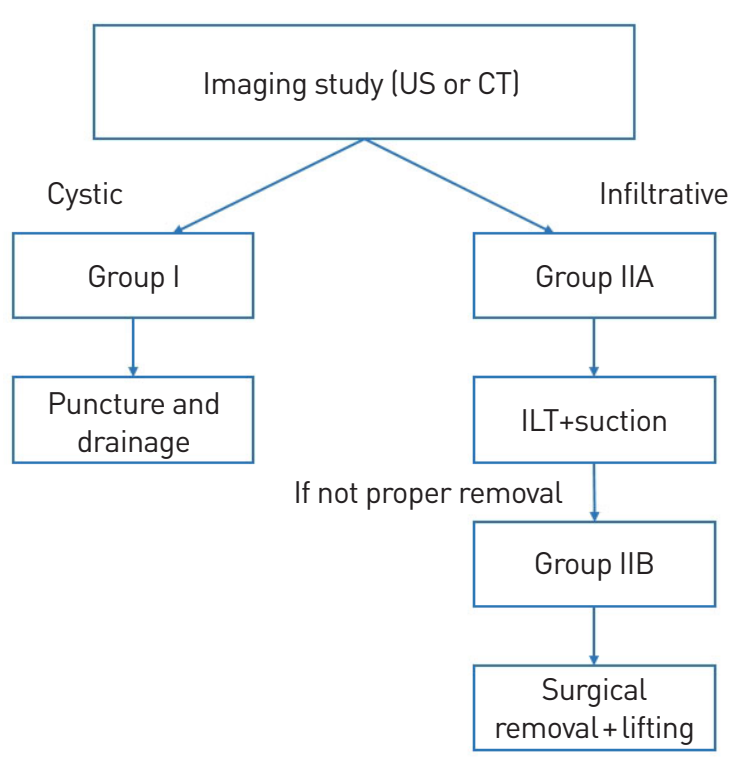

Fig. 1. Proposed algorithm for the ultrasound-based evaluation and treatment of chronic foreign body granulomas arising after filler injection. US, ultrasonography; CT, computed tomography; ILT, intralesional laser treatment. 


\section{Infiltrating group with tumescent suction after}

intralesional laser treatment (group IIA)

In the infiltrating group, which showed fine fibrosis in connective tissue on ultrasound images, we injected a tumescent solution (1,000 mL of Ringer's solution, $20 \mathrm{~mL}$ of $2 \%$ lidocaine, $1 \mathrm{~mL}$ of 1:1,000 epinephrine, and $1 \mathrm{~mL}$ of hyaluronidase). Then, using an 18-gauge needle, puncture was performed along the relaxed skin tension line, and a fiber laser (SILUET $1470 \mathrm{~nm}$ laser; WONT$\mathrm{ECH}$, Daejeon, Korea) was inserted into the lesion. The parameters of intralesional laser treatment were as follows: power, $5 \mathrm{~W}$; frequency, $500 \mathrm{~Hz}$; and energy, 100-200 J. For sensitive skin areas, such as around the eyes and the glabellar region, and in skin areas that previously received steroid injections, the intensity of the procedure was reduced. Before the procedure, patients were fully informed of the possibilities of postoperative tissue damage or burns and instructed to notify the operator if they felt too hot or nauseous [8]. The diameter of the fiber laser used was $400 \mu \mathrm{m}$, and softening of the tissue during laser treatment was used as the procedural endpoint. After intralesional laser treatment, suction was performed. However, if the intralesional laser treatment failed to remove the foreign body completely, surgical removal was performed. To minimize the resulting deformity, such as depression, a lifting procedure was performed depending on the site.

\section{Infiltrating group with surgical removal through an} inconspicuous incision (group IIB)

\section{Foreign body granuloma in the forehead: surgical removal through an anterior hairline incision}

To ensure that scars were as inconspicuous as possible, an anterior hairline incision was designed at an angle of $30^{\circ}-45^{\circ}$ from the skin surface when removing foreign body granulomas in the forehead. The forehead skin flap was elevated to about $2 \mathrm{~cm}$ above the eyebrows. Care was taken to avoid nerve damage during dissection and deformities, such as depression, after surgery. To achieve aesthetically favorable results after removal, excess skin was excised appropriately at the anterior hairline during the forehead lift procedures.

\section{Foreign body in the lower lid: surgical removal through a subciliary incision}

To ensure that scars were as inconspicuous as possible, subciliary incisions were performed to remove foreign bodies from a lower lid. A subciliary incision was performed if there was a natural crease or more frequently about $2 \mathrm{~mm}$ away from cilia. Foreign bodies were removed by dissection downward along the preseptal plane beneath the orbicularis oculi, taking care not to injury the infraorbital nerve. To achieve aesthetically favorable results after removal, orbital fat was relocated to depressions as necessary to prevent deformations, and midface lift was performed if necessary.

\section{RESULTS}

In patients with a foreign body granuloma after injection of a foreign body such as a filler, as determined by high-resolution ultrasonography, we confirmed that the foreign body exhibited a hypoechoic nodular infiltration or cyst in the dermal layer, subcutaneous fat layer, or muscle layer. Follow-up was performed for 1 to 36 months after surgery, and the average follow-up period was 15 months. Of the 35 patients included, $20 \%(\mathrm{n}=7)$ were classified into group I, $40 \%(n=14)$ to group IIA, and $40 \%(n=14)$ to group IIB. The procedures were successful in most patients, but during follow-up, inadequate removal and contour deformity were observed in four patients, of whom two patients underwent reoperation and two patients showed improvement under observation. Two group IIB patients who underwent midface lift had hematomas, which were removed, and both had symptoms such as chemosis that improved after conservative treatment. In most patients, the functional and aesthetic results were also satisfactory.

\section{Case 1}

A 38-year-old woman who had received an illegal filler injection on the forehead, temples, and cheeks about 8 years previously and filler injections 2-3 times on the cheeks at a local clinic about a year previously presented with bulging of the lateral side of the nasolabial fold. Preoperative high-resolution ultrasonography revealed several hypo-echoic lesions with relatively clear boundaries with surrounding tissues in both cheeks. The skin above the lesions was punctured with an 18-gauge needle. After puncture and drainage through manual pressure, the empty space was washed with saline to remove residual foreign matter (Fig. 2).

\section{Case 2}

A 40-year-old woman visited our hospital for intermittent swelling of the face with a duration of 5-6 years after receiving an injection of an unknown substance into the forehead and glabella about 10 years previously. Unknown foreign substances were scattered over surrounding soft tissue in the forehead and glabella areas that had similar echoic characteristics. A tumescent solution was injected around the foreign bodies in the lesion, followed by intralesional laser treatment and suctioning (Fig. 3).

\section{Case 3}

A 63-year-old woman who had received an injection of an unknown substance into the forehead and glabella about 10 years ago presented complaining of swelling in the forehead area. Computed tomography images of the facial area performed at presentation revealed foreign bodies scattered in the forehead and soft tissues of the glabellar region. After injecting tumescent solution, suction was performed, including the surrounding soft tissue, and this was followed by surgical removal because the foreign bodies were not 

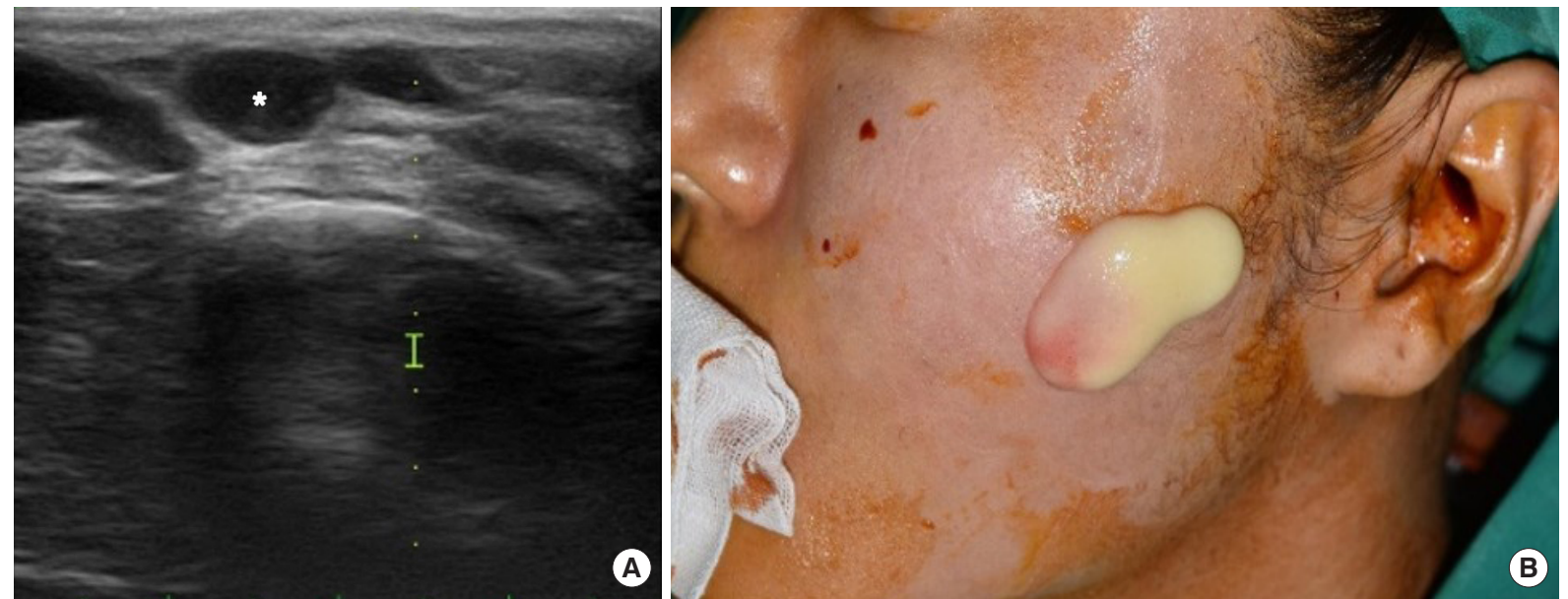

Fig. 2. A 38-year-old woman presented with visible and palpable mass lesions in the left cheek after injection of an unknown substance. (A) Intraoperative ultrasonography view of the cystic lesion (white asterisk). (B) Intraoperative view of a foreign body with gentle finger compression immediately after puncture.
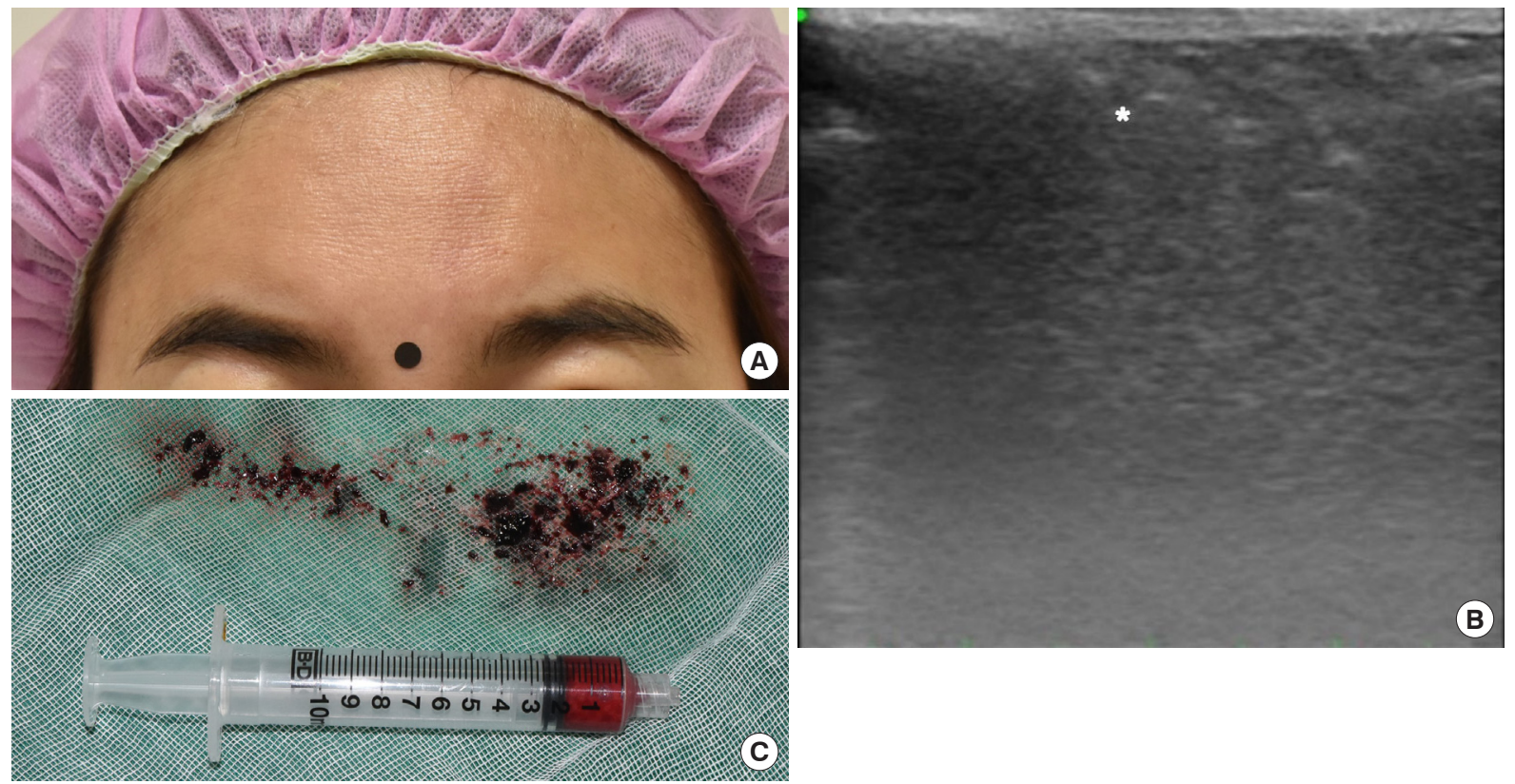

Fig. 3. A 40-year-old woman presented with visible and palpable forehead irregularities after injection of an unknown substance. (A) Photograph taken preoperatively. (B) Preoperative ultrasonography view of the facial foreign body granuloma (white asterisk). (C) Intraoperative removal of foreign body granuloma after intralesional laser treatment and suction.

completely removed. For minimally invasive removal, an anterior hairline incision was selected. The foreign body was removed evenly from under the flap based on considerations of the forehead contour (Fig. 4).

\section{Case 4}

A 72-year-old woman who had received an injection of an unknown substance into the lower eyelid for cosmetic purposes about 5 years ago presented complaining of bulging and pigmentation of both lower eyelids. Preoperative ultrasonography showed the infiltration of unknown substances in both lower eyelids. Subciliary incisions were performed to remove foreign bodies from both lower eyelids. Foreign bodies were removed by dissection downward along the preseptal and subcutaneous plane. To minimize the risk of postoperative deformities such as depression, orbital fat transposition was performed (Fig. 5). 

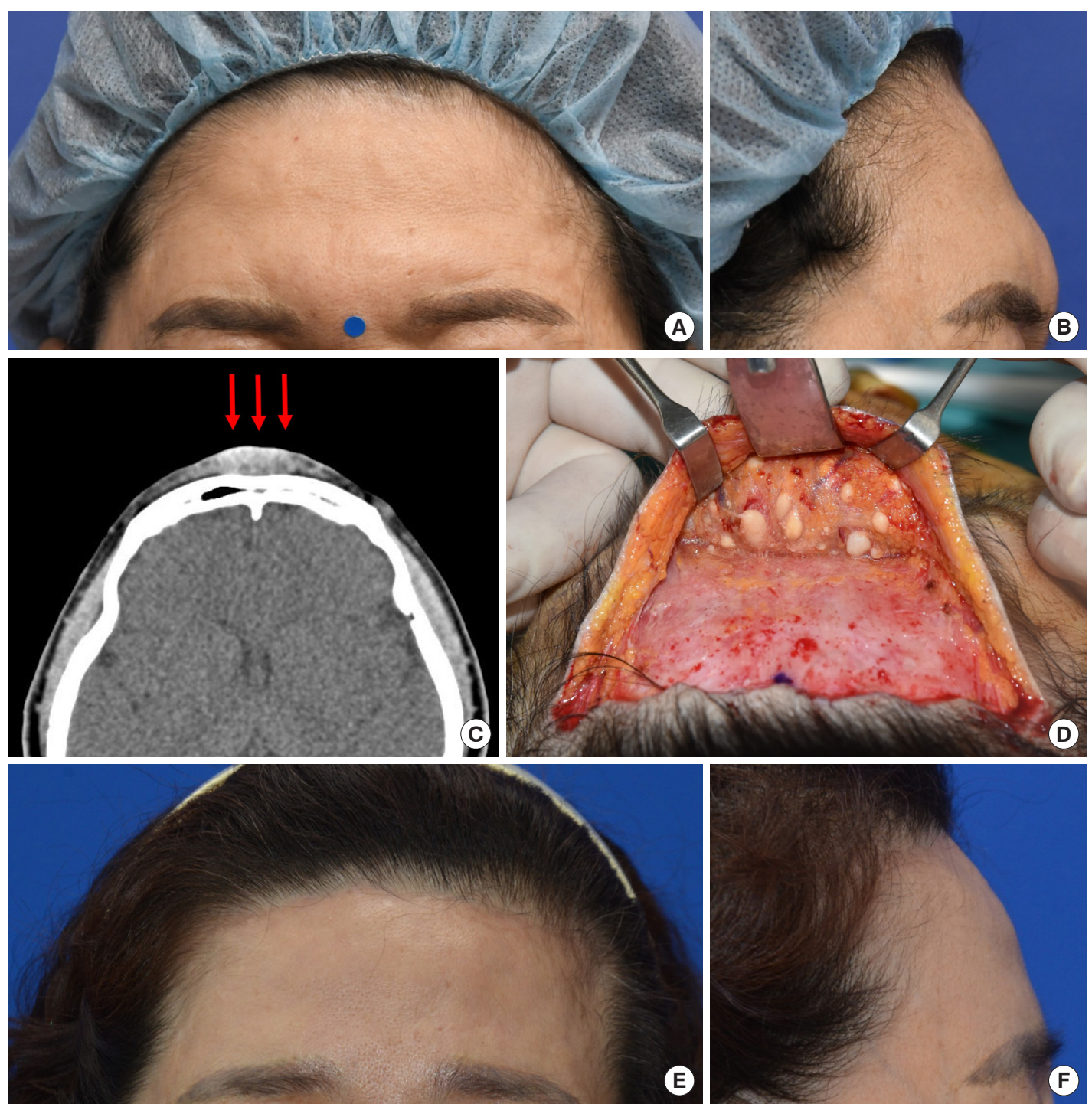

Fig. 4. A 63-year-old woman presented visible and palpable forehead irregularities after injection of an unknown substance. (A, B) Photograph taken preoperatively. (C) A preoperative axial view of computed tomography of a diffuse foreign body granuloma around the forehead (red arrows) (D) Intraoperative view of the foreign body granuloma. (E, F) Photograph taken at 10 months postoperatively.

\section{DISCUSSION}

Microinvasive techniques such as filler injection continue to spread, and reportedly, the incidence of acute side effects (e.g., edema, pain, erythema, bleeding, and ecchymosis) and delayed side effects (e.g., inflammatory or granulomatous foreign body reactions, tissue necrosis, and position shift of injected substances) is increasing [9]. Complete resection of the affected tissue may be attempted to treat irregularities of the forehead contour caused by a granulomatous foreign body reaction, but surgical removal of exposed or extensive lesions of the forehead or midface may be difficult [10]. A recent case report described improvements in the removal of foreign body granulomas with only small incision using Versajet handpieces. Versajet is a razor-thin saline jet for tissue debridement and involves reduced scarring because of the smaller incisions [7]. However, the results of these attempts have so far been variable.

Lemperle and Gauthier-Hazan [11] argued that granulomatous foreign body reactions have a latency of several months, whereas foreign body nodules occur within the initial 2-4 weeks, and that an early diagnosis and intralesional injection of triamcinolone can produce good results. The initial dose starts at $20-40 \mathrm{mg}$ every 4 weeks and can be increased if no effect is observed within 2-4 weeks; it was reported that the dose was increased to $160 \mathrm{mg}$ in 6 months in African American and Asian patients. To avoid skin atrophy, combination therapies such as 5 -fluorouracil $(50 \mathrm{mg} / \mathrm{mL})$ and pulsed laser treatment are effective. Intralesional steroid injection is highly effective as an initial treatment, but in the authors' experience, in most patient groups, non-surgical treatments, in- 

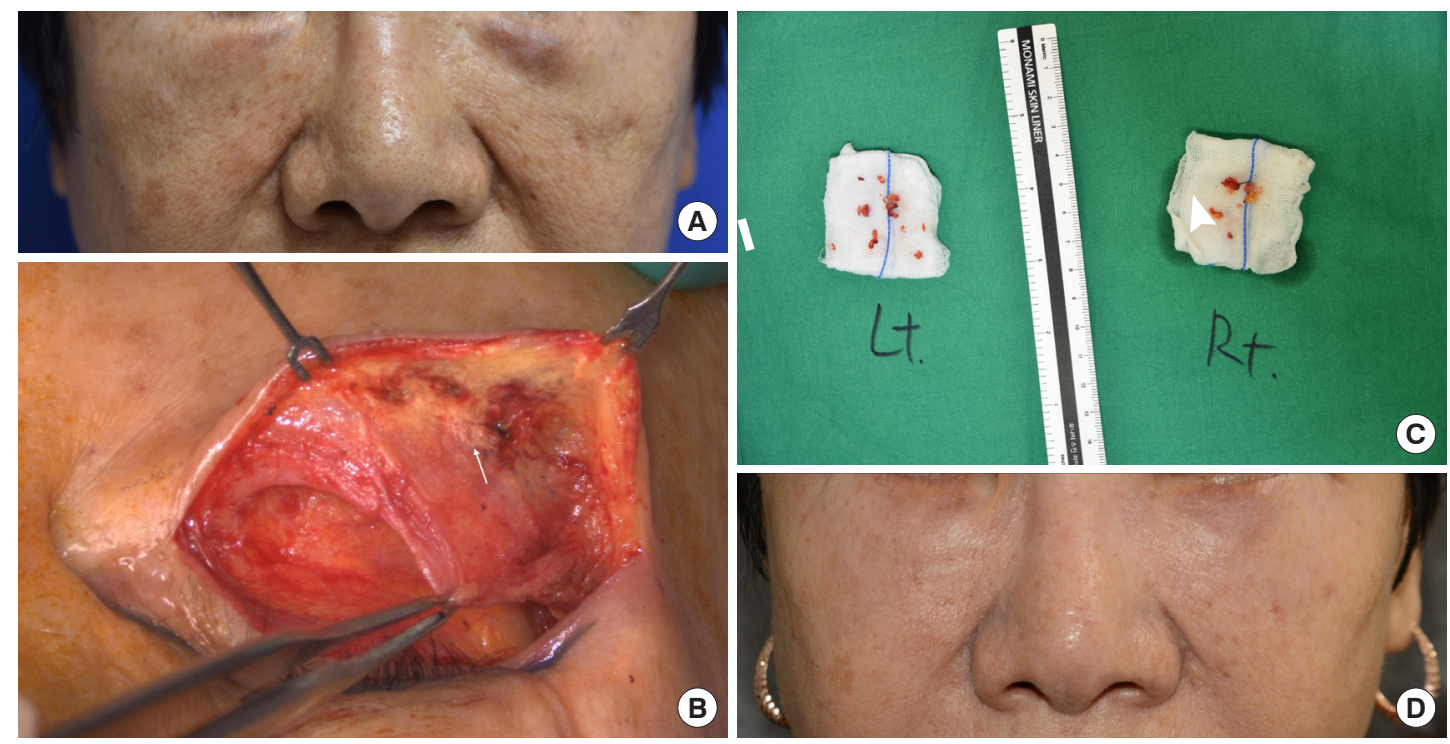

Fig. 5. A 72-year-old woman presented with bulging and pigmentation of both lower eyelids after injection of an unknown substance. (A) Photograph taken preoperatively. (B) Intraoperative view of a foreign body granuloma on the lower eyelid (white arrow) (C) Intraoperative removal of the foreign body granuloma. (D) Photograph taken at 6 months postoperatively.

cluding steroids, usually have only temporary effects. Furthermore, it has been frequently reported that additional surgical treatment is necessary due to inflammation, infection, or repeated cycles of deterioration and improvement.

It is important to determine the causative agent and the condition of soft tissue in patients that present with filler complications, and the importance of ultrasonography in this context has been emphasized by various authors. Schelke et al. [12] reported 72 patients who had been injected with fillers into their faces and found that hydrophilic fillers showed non-echoic or hypo-echoic tendencies and permanent fillers showed the same echoes as the surrounding tissues on 13-20 MHz ultrasound examinations. Another report described good results in 20 patients with foreign body granulomas after filler injections who were evaluated using ultrasonography $[13,14]$. In this study, based on imaging findings, lesions were classified as cystic or infiltrative. Ultrasonography also enabled determination of the lesion extent, location, and depth, thereby facilitating treatment planning and reducing unnecessary incisions. In our cases, patients with cystic granulomas were treated by puncture, manual compression, and drainage. This allowed us to avoid unnecessary incisions in patients with cystic granulomas treated by puncture, manual compression, and drainage.

Schelke et al. [8] reported good results after intralesional laser treatment using an 810-nm diode laser or a 1,470-nm diode laser targeting hemoglobin and water in the blood, respectively, in 242 of 590 patients with filler complications. Subsequently, the liquefied permanent filler was punctured and drained. A recent report described a case of clinical improvement after surgical removal of foreign bodies using liposuction in a patient with facial edema as- sociated with multiple skin-colored nodules in forehead and both cheeks after the injection of an unknown substance 10 years previously [5]. Grippaudo et al. [15] and Bassetto et al. [16] tried to remove siliconomas from the face, chest, and buttocks under ultrasound guidance by liposuction and reported satisfactory cosmetic and functional improvements in most patients. In this study, in the infiltrating group, after intralesional laser treatment, suction was performed that included some surrounding soft tissue to enhance the effectiveness. We used a 1,470-nm diode laser. Unlike other laser wavelengths, the 1,470-nm wavelength is well-absorbed by both water and fat. Therefore, this wavelength does not require highpower energy during operation and it saves operation time and enables the prevention of undesirable damage.

Surgical removal was performed in the infiltrating group when a foreign body was insufficiently removed by suction combined with intralesional laser treatment or it was expected to cause cosmetic problems such as depression, wrinkles, and contour irregularity. There was a report about good results after surgical resection for four patients with foreign body granulomas after filler injection into the face [17]. However, simple resection is limited as it results in scars, depressions, and deformities; thus, simple surgical resection of multiple granulomatous facial lesions is inadvisable. Therefore, to ensure that the scars were as inconspicuous as possible, an anterior hairline incision or subciliary incision was used. To minimize postoperative deformities, such as depression and wrinkles, forehead lift or midface lift was performed concomitantly if necessary.

In this study, the best results were obtained by accurately identifying lesion characteristics by imaging studies in patients with widespread facial granulomas for which it was difficult to determine the 
surgical method. It is important to estimate the characteristics of unknown substances in facial foreign body granulomas for determining the best method of treatment. Based on our experience, we suggest a treatment algorithm using imaging studies. Most patients can be classified as cystic or infiltrating according to the ultrasound pattern. Good results were obtained by puncture and drainage in the cystic group. In the infiltrating group, patients first received suction after intralesional laser treatment; if insufficient, surgical removal through an inconspicuous incision was additionally performed. After intralesional laser treatment, the lesions were removed by suction, and when suction was insufficient or the lesions were extensive, surgical removal was considered. In cases of direct lesion resection and removal, the incision line was made inconspicuous to avoid extensive scarring and the formation of depression. In cases involving the forehead and glabella, we made incision lines via the anterior hairline, and when facial granulomas were in the midface, we made subciliary incisions. In addition, skin depressions and facial wrinkles were minimized by various facial lifting procedures with the concurrent removal of foreign body granulomas. Thus, this report suggests minimally invasive and cosmetically more favorable methods for the treatment of facial foreign body granulomas that are difficult to treat clinically.

\section{NOTES}

\section{Conflict of interest}

No potential conflict of interest relevant to this article was reported.

\section{Ethical approval}

The study was approved by the Institutional Review Board of Chungnam National University Hospital (IRB No. CNUH 2019-10-063) and performed in accordance with the principles of the Declaration of Helsinki.

\section{Patient consent}

The patients provided written informed consent for the publication and the use of their images.

\section{ORCID}

Jaeik Choi

Geonil Ko

Hyeokjae Kwon

Yooseok Ha

Sunje Kim

Hyunwoo Kyung

Sang-Ha Oh

\section{REFERENCES}

1. Neuber F. Fat transplantation. Chir Kongr Verhandl Dsch Gesellch Chir 1893;20:66.

2. Rohrer TE. Soft tissue filler substances. Curr Probl Dermatol 2001;13: 54-60.

3. Han JH, Lee JH, Lee JY, et al. A case of foreign body granuloma after squalene injection by non-dermatologists. Korean J Dermatol 2016;54: 671-2.

4. Park TH, Seo SW, Kim JK, et al. 120 Cases of facial foreign body granuloma. J Korean Soc Plast Reconstr Surg 2010;37:233-8.

5. Oh SJ, Park BJ, Ko JY, et al. Foreign body granuloma after filler injection treated with liposuction. Korean J Dermatol 2017;55:533-5.

6. Hong JY, Suh JH, Ko EJ, et al. Chronic, intractable nodules after filler injection successfully treated with a bipolar radiofrequency device. Dermatol Ther 2017;30:e12400.

7. Choi M, Son KM, Choi WY, et al. Usefulness of the Versajet hydrosurgery system for the removal of foreign body granuloma. Arch Plast Surg 2017;44:352-3.

8. Schelke LW, Decates TS, van der Lugt CIM, et al. Intralesional laser treatment for dermal filler complications. Plast Reconstr Surg 2018;141: $1361-9$.

9. Bae JM, Kim MY, Kang H, et al. The clinicopathologic study of foreign body granuloma induced by injection of filler. Korean J Dermatol 2007; 45:255-61.

10. Rudolph CM, Soyer HP, Schuller-Petrovic S, et al. Foreign body granulomas due to injectable aesthetic microimplants. Am J Surg Pathol 1999;23:113-7.

11. Lemperle G, Gauthier-Hazan N. Foreign body granulomas after all injectable dermal fillers: part 2. Treatment options. Plast Reconstr Surg 2009;123:1864-73.

12. Schelke LW, Van Den Elzen HJ, Erkamp PP, et al. Use of ultrasound to provide overall information on facial fillers and surrounding tissue. Dermatol Surg 2010;36 Suppl 3:1843-51.

13. Cassuto D, Pignatti M, Pacchioni L, et al. Management of complications caused by permanent fillers in the face: a treatment algorithm. Plast Reconstr Surg 2016;138:215e-227e.

14. Cassuto D, Marangoni O, De Santis G, et al. Advanced laser techniques for filler-induced complications. Dermatol Surg 2009;35 Suppl 2:168995.

15. Grippaudo FR, Spalvieri C, Rossi A, et al. Ultrasound-assisted liposuction for the removal of siliconomas. Scand J Plast Reconstr Surg Hand Surg 2004;38:21-6.

16. Bassetto F, Abatangelo S, Masetto L, et al. Ultrasound-assisted liposuction as a safe and effective method for the removal of siliconomas. Aesthetic Plast Surg 2012;36:220-2.

17. Wolfram D, Tzankov A, Piza-Katzer H. Surgery for foreign body reactions due to injectable fillers. Dermatology 2006;213:300-4. 